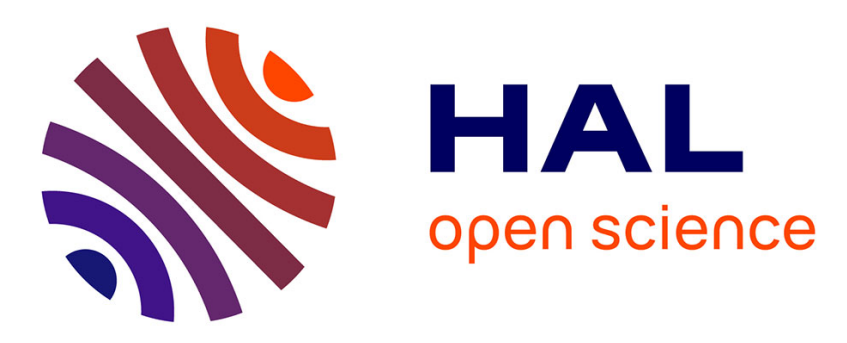

\title{
Stabilizing Vortices in Interacting Nano-Objects: A Chemical Approach
}

\author{
Lise-Marie Lacroix, Sébastien Lachaize, Florian Hüe, Christophe Gatel, \\ Thomas Blon, Reasmey Phary Tan, Julian Carrey, Bénédicte Warot-Fonrose, \\ Bruno Chaudret
}

\section{- To cite this version:}

Lise-Marie Lacroix, Sébastien Lachaize, Florian Hüe, Christophe Gatel, Thomas Blon, et al.. Stabilizing Vortices in Interacting Nano-Objects: A Chemical Approach. Nano Letters, 2012, 12 (6), pp.3245-3250. 10.1021/nl3012616 . hal-03156654

\section{HAL Id: hal-03156654 https://hal.science/hal-03156654}

Submitted on 2 Mar 2021

HAL is a multi-disciplinary open access archive for the deposit and dissemination of scientific research documents, whether they are published or not. The documents may come from teaching and research institutions in France or abroad, or from public or private research centers.
L'archive ouverte pluridisciplinaire HAL, est destinée au dépôt et à la diffusion de documents scientifiques de niveau recherche, publiés ou non, émanant des établissements d'enseignement et de recherche français ou étrangers, des laboratoires publics ou privés. 


\section{Stabilizing vortices in interacting nano-objects : a}

\section{chemical approach}

Lise-Marie Lacroix*, ${ }^{1}$ Sébastien Lachaize, ${ }^{1}$ Florian Hue,,${ }^{2,3}$ Christophe Gatel, ${ }^{4}$ Thomas Blon, ${ }^{1}$ Reasmey P. Tan, ${ }^{1}$ Julian Carrey, ${ }^{1}$ Bénédicte Warot-Fonrose, ${ }^{4}$ Bruno Chaudret ${ }^{1}$

1. Université de Toulouse, INSA, UPS, LPCNO (Laboratoire de Physique et Chimie des Nano-Objets), F-31077 Toulouse, France; CNRS; UMR 5215 ; LPCNO, F-31077 Toulouse, France

2. Department of Materials Science and Metallurgy, University of Cambridge, Pembroke Street, Cambridge, CB2 3QZ, United-Kingdom.

. 3. Present address : Université de Rouen, UMR 6634 CNRS, F-76801 Saint-Etienne du Rouvray, France

4. CEMES-CNRS, 29 rue J. Marvig, BP4347, 31055 Toulouse, France

* corresponding author : email, 1mlacroi@ insa-toulouse.fr; phone +33561559652, fax +33561559697

RECEIVED DATE (to be automatically inserted after your manuscript is accepted if required according to the journal that you are submitting your paper to)

ABSTRACT : We report a chemical method to prepare metallic Fe porous nanocubes. The presence of pores embedded inside the cubes was attested by electron tomography. Thanks to electronic holography and micromagnetic simulations, we show that the presence of these defects stabilizes the vortices in assembly of interacting cubes. These results open new perspectives towards magnetic vortex 
stabilization at relatively low cost for various applications (microelectronics, magnetic recording or biological applications).

KEYWORDS. Magnetic nanoparticles; bcc-Fe; Porous nano-objects; Vortex stabilization; Electron holography; Electron tomography

\section{INTRODUCTION :}

Magnetic nanoparticles (NPs) are objects of choice for multiple purposes such as magnetic recording, ${ }^{1,2}$ which requires high storage density, ${ }^{3,4}$ and biomedical applications, ${ }^{5}$ such as MRI contrast agents, ${ }^{6}$ or therapeutic hyperthermia media. ${ }^{7,8}$ Although single-domain magnetic materials are usual targets for these applications, ${ }^{9,10}$ vortex configurations, which arise at the transition between multidomain and single-domain materials, could be of particular interest for dedicated purposes. ${ }^{11}$ Indeed, vortices are characterized by their polarity, i.e. the direction of magnetization in the core (up/down), and their chirality, i.e. the direction of rotation around the vortex core (clockwise or counterclockwise). ${ }^{12}$ As the vortex polarity and chirality are independent, four magnetic states could be obtained (Supplementary Scheme 1), thus opening the perspective of two-bit-per-dot media for magnetic storage, providing they can be manipulated. ${ }^{13,14,15}$ Additionally, the excitation of vortices by a spinpolarized current leads to high-quality radio-frequency oscillators, even in the absence of external magnetic field, which is very promising for potential applications. ${ }^{16,17,18}$ Finally, vortices have recently been reported as presenting high potentiality for biomedical applications since they combine high saturation magnetization, required for bioapplications, with negligible remanence magnetization, which is a key feature for stabilizing ferrofluids against aggregation phenomena. ${ }^{19}$

However, in all of these applications, the stabilization of vortices in presence of magnetic dipolar interactions (for closely packed nanostructures or highly contentrated ferrofluids) is crucial. We have previously reported that an isolated $30 \mathrm{~nm}$ Fe nanocube exhibits a vortex state. ${ }^{20}$ However, ferromagnetic coupling appeared between self-assembled nanocubes, leading to the disappearance of 
individual vortex in favor of more stable flux closure configurations across several cubes. ${ }^{20}$ For dipoles of $\mathrm{Fe}_{3} \mathrm{O}_{4}$ nanorings, theoretical works have also shown a magnetic coupling leading to the disappearance of vortices. ${ }^{19}$ In order to overpass this limitation, one should improve the stability of the vortex within a single object. Two options may be considered : an increase of the object size, which will be a drawback for applications, or the introduction of internal vacancies and defects within small object which could pin the vortex core. ${ }^{21,22}$ Up to now, the experimental preparation of such artificial pinning sites (defects, holes) requires costly multi-step patterning processes. ${ }^{21,23}$ An alternative method for the preparation of magnetic objects with embedded defects could be liquid-phase chemical reactions.

Though synthesis of magnetic nanoparticles with high magnetization values has been a longsought goal, researches were mainly focused on defect-free particles. ${ }^{9}$ Except from hollow $\mathrm{Fe}_{3} \mathrm{O}_{4}$ spheres or rods, ${ }^{19,24}$ synthesis of metallic nanoparticles with pores embedded has never been reported. Based on our know-how in the size and shape control of iron nanoparticles, ${ }^{25}$ we have succeeded in the growth of porous particles. Our method is based on a mediated-growth which proceeds through three distinct steps: i) nucleation; ii) growth/coalescence iii) growth/ripening. The control of the growth/coalescence step has recently received lots of interest yielding nanoparticles with unique features. ${ }^{26,27}$ Here its control allows us to force the presence of holes within nanocubes.

\section{METHOD :}

Synthesis of porous cubes.

A colorless solution of lauric acid (300 mg, $1.5 \mathrm{mmol}$, 1.5 equivalent per mole of iron) in mesitylene $(10 \mathrm{~mL})$ was added to a green solution of $\left\{\mathrm{Fe}\left[\mathrm{N}\left(\mathrm{SiMe}_{3}\right)_{2}\right]_{2}\right\}_{2}(376 \mathrm{mg}, 0.5 \mathrm{mmol})$ in mesitylene $(5 \mathrm{~mL})$ under Ar atmosphere. The mixture was manually stirred and its colour immediately turned to yellow. One minute later, a solution of dodecylamine (370 mg, $2.0 \mathrm{mmol}, 2.0$ eq.) in mesitylene $(5 \mathrm{~mL})$ was added to it. The mixture started to darken and it became black after 10 min of magnetic stirring at room temperature. The solution was then put under 3 bars of $\mathrm{H}_{2}$ and let to react in an oil bath at $150^{\circ} \mathrm{C}$ for $48 \mathrm{~h}$. See Supplementary material for further details. 
Characterization of the NPs. Microscopy samples were prepared by deposition of a diluted colloidal solution drop on a carbon coated copper grid and observed on a JEOL-1011 for Bright Field Transmission Electronic Microscopy (TEM) and JEOL-2100F field emission microscope for High Resolution-TEM (HRTEM) working with $100 \mathrm{kV}$ and $200 \mathrm{kV}$ respectively. In the majority of cases, size histograms were obtained by manual counting process over at least 100 particles, using ImageJ software. ${ }^{28}$ Size distributions have been fitted by Gaussian law; the results are expressed by the calculated mean size and standard deviation $\sigma$. Magnetic studies were carried out on powder samples by SQuID (MPMS Quantum Design 5.5) and the iron state and environment were analysed by Mössbauer spectroscopy (WISSEL, ${ }^{57}$ Co source). Samples were prepared into the glove box and extreme care was taken to avoid oxidation during transfer to the apparatus. Flame sealed glass tubes of powder were prepared under argon to determine the iron composition from microanalysis measurements performed by ICP (Inductively Coupled Plasma).

Electron Tomography To study the morphology of these Fe cubes, we use electron tomography in scanning transmission electron microscopy (STEM) mode using High Angle Annular Dark Field (HAADF) detector. This technique is well suited to obtain size and morphology information of crystalline materials without suffering from Fresnel fringes and diffraction contrast. A full 3D analysis is then possible. We acquire HAADF-STEM tilt series on a FEI Tecnai F20 microscope fitted with a Fischione high-tilt tomography holder. The holder is designed to allow a $+/-80^{\circ}$ tilt without being limited by the pole piece gap of the microscope. The only restriction comes from the specimen itself and its support (400 mesh grid, in our case). We acquired two tilt series with 2 different camera lengths. The first tilt series was taken between $-66^{\circ}$ and $+70^{\circ}$ with an angular step of $2^{\circ}$ between each acquisition. The tilt maximum leads to a missing wedge of information that induces an artefact of elongation in the $\mathrm{z}$ direction. In our case the global missing wedge is $44^{\circ}$ and induces an elongation with a ratio of 1.1 in $\mathrm{z}^{29}$ The second tilt series was acquire with a $-45+56$ tilt range which leads to a strong elongation in $\mathrm{z}$ (ratio of 1.35) but the camera length was more appropriate and the contrast of the projections as well as the quality of the 3D object were improved. In both cases, the projections were sorted out under inspect3D software and aligned by cross-correlations, using an appropriate spatial filter.

Electron Holography The structures of the different layers and interfaces were investigated by transmission electron microscopy (TEM) both in conventional and in high-resolution mode using a FEI Tecnai-F20 microscope fitted with a spherical aberration (Cs) corrector (CEOS) whose point resolution is $0.12 \mathrm{~nm}$. We use off-axis $\mathrm{EH}$ in the same microscope to study the remnant magnetic state of the Fe porous nanocubes at room temperature. Holograms were digitally acquired with switching off the conventional objective lens and using the first transfer lens of the Cs corrector and the projectors to 
reach a maximum magnification of about x300 000. The phase images were obtained by reconstruction the hologram using Fourier transformation and removing any additional phase shift using a reference hologram obtained in vacuum. The spatial resolution of the phase images is determined by the fringe spacing, which depends on the voltage applied to the biprism $(2,3 \mathrm{~nm}$ at $135 \mathrm{~V})$ and the diameter of the spatial-frequency filter $(6 \mathrm{~nm})$ applied in the Fourier space when reconstructing the holograms. The magnetic contribution to the phase shift was extracted by recording holograms in two opposite directions (sample up / down).

Micromagnetic calculations We used the three-dimensional version of the micromagnetic code OOMMF to determine the magnetic configuration in the samples. ${ }^{30}$ The 3-D nanocube shapes and sizes introduced for the calculations are deduced from the 2-D TEM images with the assumption that the Fe cubes are perfectly cubic. Each iron nanocube is discretized in 3-D cells with $4 \mathrm{~nm}$ side length. We used bulk magnetic parameters for iron: saturation magnetization $\mathrm{M}_{\mathrm{S}}=1.72 \times 10^{6} \mathrm{~A} / \mathrm{m}$, exchange stiffness $\mathrm{A}=$ $2.1 \times 10^{-11} \mathrm{~J} / \mathrm{m}$ and cubic anisotropy constant $\mathrm{K}_{1}=4.8 \times 10^{4} \mathrm{~J} / \mathrm{m}^{3}$. A damping parameter of $\alpha=0.5$ was used to reach equilibrium remnant state rapidly. To fit the simulations with the experimental procedure, we applied magnetic fields of the same amplitude and direction as were applied experimentally. The nanoparticles have been deposited onto the TEM grid in presence of a $250 \mathrm{mT}$ magnet, generating a field along [001] direction. Then, the objective lens, though switched off, induces a residual magnetic field of ca. $20 \mathrm{mT}$ parallel to the optic axis due to the remanence of polar pieces. Thus, we included in the simulations this small magnetic field parallel to [001]. Electron holography provides a 2-D induction map of the magnetic nanocubes. In order to compare our simulations with experimental results we add the magnetization and total field vector coordinates in each cell to obtain a 3-D induction map. Then, the calculated induction vectors are averaged along the $\mathrm{z}$ direction to obtain a 2-D map of the simulated induction integrated along the sample thickness.

\section{RESULTS \& DISCUSSION :}

The reduction of $\left(\mathrm{Fe}\left\{\mathrm{N}\left[\mathrm{Si}\left(\mathrm{CH}_{3}\right)_{3}\right]_{2}\right\}_{2}\right)_{2}$ in mesitylene under a dihydrogen atmosphere at $150^{\circ} \mathrm{C}$ leads to nanoparticles of $1.5 \mathrm{~nm}$ of diameter. ${ }^{31}$ Adding surfactants with a long alkyl chain and a polar head $\left(-\mathrm{NH}_{2},-\mathrm{COOH}\right)$ to the initial mixture has several effects: i) they stabilize the nanoparticles formed,

(ii) they react with the precursor to form more stable species such as iron (II) carboxylate complexes and

(iii) at large concentration, they tend to self-organize leading to a mediated-growth. ${ }^{25}$ For acid:amine 
ratio below 1.4:2 equivalents (eq.) per mole of iron, nano-objects grow in an isotropic environment, leading after 2 days of reaction to spherical nanoparticles with mean diameters ranging from 1.5 to $9 \mathrm{~nm}$ (Supplementary Figure S1).

For acid:amine ratio of 1.5:2 eq., self-assemblies of surfactants are observed concomitant with the growth of cubic nanoparticles (Supplementary Figure S2). These self-assemblies seem a prerequisite for the synthesis of cubes through a mediated-growth. By modifying the alkyl chain length of surfactants from 16 to 12 carbons, we could tune this mediated-growth. Polydisperse cubes $(42 \mathrm{~nm}, \sigma=16 \mathrm{~nm})$ were obtained after 2 days of reaction with C12 surfactants (Figure 1a, Supplementary Figure S3). Within these objects, regions with brighter contrast are observed in transmission electron microscopy (TEM, Figure 1a) and in high resolution TEM (HRTEM, Supplementary Figure S4). Such contrast could be due to local variations of thickness or of material. However, metallic Fe was the only material detected by HRTEM, thus, the thickness variation could more likely arise from local defects at the surface or embedded within the cubes.

Since TEM only provide a two-dimensional (2D) projection of the objects, electron tomography has been performed in order to determine more precisely the localization of these defects. Tomography leads to a complete 3D reconstruction of the objects based on a series of 2D TEM images acquired by High Angle Annular Dark Field Scanning TEM (HAADF-STEM) from different tilting angles. ${ }^{32}$ An $x y$ orthoslice of the 3D view of packed nanocubes is presented in Figure 2a. The presence of darker regions, corresponding to the voids, is clearly visible. Such inversion of contrast compared to previous TEM images (Figure 1a) is due to the HAADF signal, which is directly related to the sample thickness. In order to extract quantitative information on pores, an additional manual segmentation is performed. ${ }^{33}$ Gray levels are segmented all along the reconstruction volume in order to reveal the edges of the cubes and pores. After such treatment, 3D visualisation is obtained, pores being represented in light blue, surfaces of cubes by a violet envelope (Figure 2b-c, Supplementary Video 1). The presence of internal pores is clearly attested, the defects being in a large majority (>90\%) embedded within the cube. 
The presence of internal defects within cubes rises the question of their formation and nature. Below a critical size of $30 \mathrm{~nm}$, no defects are observed while above, more than $90 \%$ of cubes exhibit holes. A strong correlation between the lateral size of cubes and the number of defects could be seen, the bigger the cubes, the more defects are embedded (Supplementary Figure S5). A kinetic study was performed by monitoring the reaction, combining iron precursor with 1.5:2 eq. of acid:amine. This reaction was equally distributed into 4 Fisher-Porter bottles, let to react at $150^{\circ} \mathrm{C}$ for $2 \mathrm{~h}, 6 \mathrm{~h}, 24 \mathrm{~h}$ and $48 \mathrm{~h}$ respectively (Figure 1). Anisotropic nanoparticles with mean lateral sizes of $10 \times 6 \mathrm{~nm}$ are observed after $2 \mathrm{~h}$ of reaction. After $6 \mathrm{~h}$, this anisotropic shape is retained but facets start to appear. Coalescence of cubes through edges leads to the presence of large octapods $(18 \mathrm{~nm}, \sigma=5 \mathrm{~nm})$ and embedded pores. Finally, after $48 \mathrm{~h}$, large and polydisperse porous cubes (42 nm, $\sigma=25 \mathrm{~nm}$ ) are obtained. This monitoring, based on TEM images, seems fairly similar to the one previously reported with C16 surfactants. ${ }^{25}$ Thus a similar growth mechanism, based on successive nucleation, growth-coalescence and growth-ripening steps, seems to control the synthesis of these cubes. Ripening appears after $6 \mathrm{~h}$, as revealed by the faceting of anisotropic objects, in agreement with the observation for C16 surfactants. ${ }^{25}$ However, coalescence does not end at the appearance of ripening but last at least $24 \mathrm{~h}$. Such variation in the coalescence step duration could explain the difference in cube sizes $(21 \mathrm{~nm}$ for C16, up to $80 \mathrm{~nm}$ for C12) and the presence of defects embedded within the cubes. The reason for that extent is not known yet, but it could be linked to the difference in the stability of cube edges, due to a lower steric hindrance for C12 compared to C16 alkyl chains. Though it will be difficult to conclude on the chemical species trapped within pores, their size distribution, extracted from the projected lateral size observed by TEM, give some clues (Supplementary Figure S6). Indeed, the minimum pore size is above $4 \mathrm{~nm}$, i.e. twice the surfactant alkyl chain length. Since the defects are formed during a coalescence process, we propose that the cavities are filled with surfactants, which would explain this minimum size and the facets observed, $\{100\}$ faces being stabilized by acid. ${ }^{25}$ 
Magnetic characterization performed on raw powders at $2 \mathrm{~K}$ and $300 \mathrm{~K}$ reveals that these porous nanocubes exhibit a magnetization of respectively 220 and $216 \mathrm{Am}^{2} / \mathrm{kg}_{\mathrm{Fe}}$ close to the bulk value of bccFe and are ferromagnetic at room temperature $\left(\mu_{0} \mathrm{H}_{\mathrm{C}}=15 \mathrm{mT}\right.$, Supplementary Figure S7). The local study of the magnetic behaviour of these cubes has been performed by electron holography (EH). ${ }^{32} \mathrm{Off}-$ axis EH allows measuring the phase shift of the high-energy electron wave that has passed through a material. ${ }^{34}$ Since the phase shift is sensitive to electric and magnetic fields in the sample, local magnetic properties in nanoscale materials can be mapped after having isolated the magnetic part. The nanocubes observed have been deposited onto the TEM grid in presence of a $250 \mathrm{mT}$ magnet, generating a field along [001] direction. As expected from the size of the object above the single-domain/vortex transition $(\sim 28 \mathrm{~nm}),{ }^{11,20}$ isolated nanocubes exhibit a vortex state (Supplementary Figure S8). Perturbations of the vortex core are observed at the vicinity of pores, but the out-of-plane component of the magnetization is still clearly observed. The induction state in interacting nanocubes could also be determined (Figure 3). Contrarily to the observation previously performed on interacting $30 \mathrm{~nm}$ nanocubes ${ }^{20}$ vortices are retained within each cube presenting defects. Such a peculiar magnetic configuration could be reproduced by micromagnetic calculations (OOMMF code). ${ }^{35}$ In order to address the stability question of the vortices, we have compared the numerical simulation obtained for defect free (Figure 3c) and porous (Figure 3d) interacting cubes. Porous objects have been simulated by introducing voids within the cubes. The lateral sizes and in-plane position of the defects were extracted from TEM images. Their height was extrapolated from a cubic shape since mainly isotropic defects are observed by tomography. Finally the out-of-plane position of the defects was arbitrary taken within the cubes, equally distributed apart from the median position. Since the position of isotropic defects was reported as not affecting the vortex nucleation field, ${ }^{36}$ we did not explore the effect of the $\mathrm{z}$ position on the stability of the vortices. While a flux closure state is observed for arrangements of defect-free objects (Figure 3c), vortices are observed for porous cube as evidenced in the integrated induction map (Figure 3d) and in the 3D magnetic moment cartography (Figure 4). Thus, in presence of internal defects, vortices become the configuration of minimum energy, due to the overall reduction of the exchange energy. ${ }^{23,37}$ However, 
most of the results previously reported have been handled on permalloy nanodisks with single lithography-defined defect of same thickness than the object. ${ }^{21,23,36}$ In such cases, the vortex core is stabilized within the void, resulting in the total disappearance of the out-of-plane singularity, and thus, to an onion configuration rather than a vortex configuration. In our case, where multiple voids are smaller and randomly distributed within the materials, the vortex core is still observed within the cubes. Thus, the detailed mechanism of vortex stabilization could be more complicated and would benefit from deeper investigations with cross correlated electronic tomography-holography measurements and micromagnetic simulations.

In conclusion, a fine tuning of the growth environment and the use of appropriate aliphatic surfactants allowed us to synthesize quantitatively Fe nanocubes above 30nm and incorporating voids. The presence of internal defects embedded within cubes has been characterized by electron tomography. These defects have been for the first time evidenced as stabilizing vortex magnetic states within nanoparticle interacting assemblies. In this respect, we provided experimental proof for the potential interest of metallic porous cubes as stable vortices structures. These vortices are particularly important for advanced fundamental studies and might eventually become new building blocks for dedicated applications (spintronics, magnetic recording, nanomedecine). Subsequent optimizations need however to be undertaken, such as stabilizing defects within smaller objects, reducing the overall size dispersity of the Fe cubes and controlling their self-assembly on large 2D areas.

ACKNOWLEDGMENT: L.-M.L thanks the French Ministry of "National Education and Research" for MENRT grant. The authors thank the METSA and ESTEEM networks for microscopy studies, Alain Mari for magnetic measurements and Marc Respaud for fruitful discussions. 
SUPPORTING INFORMATION AVAILABLE: (1) Experimental procedure (General synthesis, NP characterization, Electronic Tomography and Holography experiments, Micromagnetic simulations). (24) TEM images of nanoparticles obtained under various experimental conditions (ligands, temperature, concentration). Characterization of porous cubes by $(5,8)$ HRTEM (6) SEM, (7) TEM. (9) Magnetic characterization of bcc-Fe porous NPs $(\mathrm{M}(\mathrm{H})$ at 2 and 300K); (10) Electron holography on a single porous nanocube; (11) 3D map of the magnetic moment obtained by micromagnetic simulation within an assembly of porous nanocubes. A video of the electron tomography reconstruction is also available.

FIGURES 


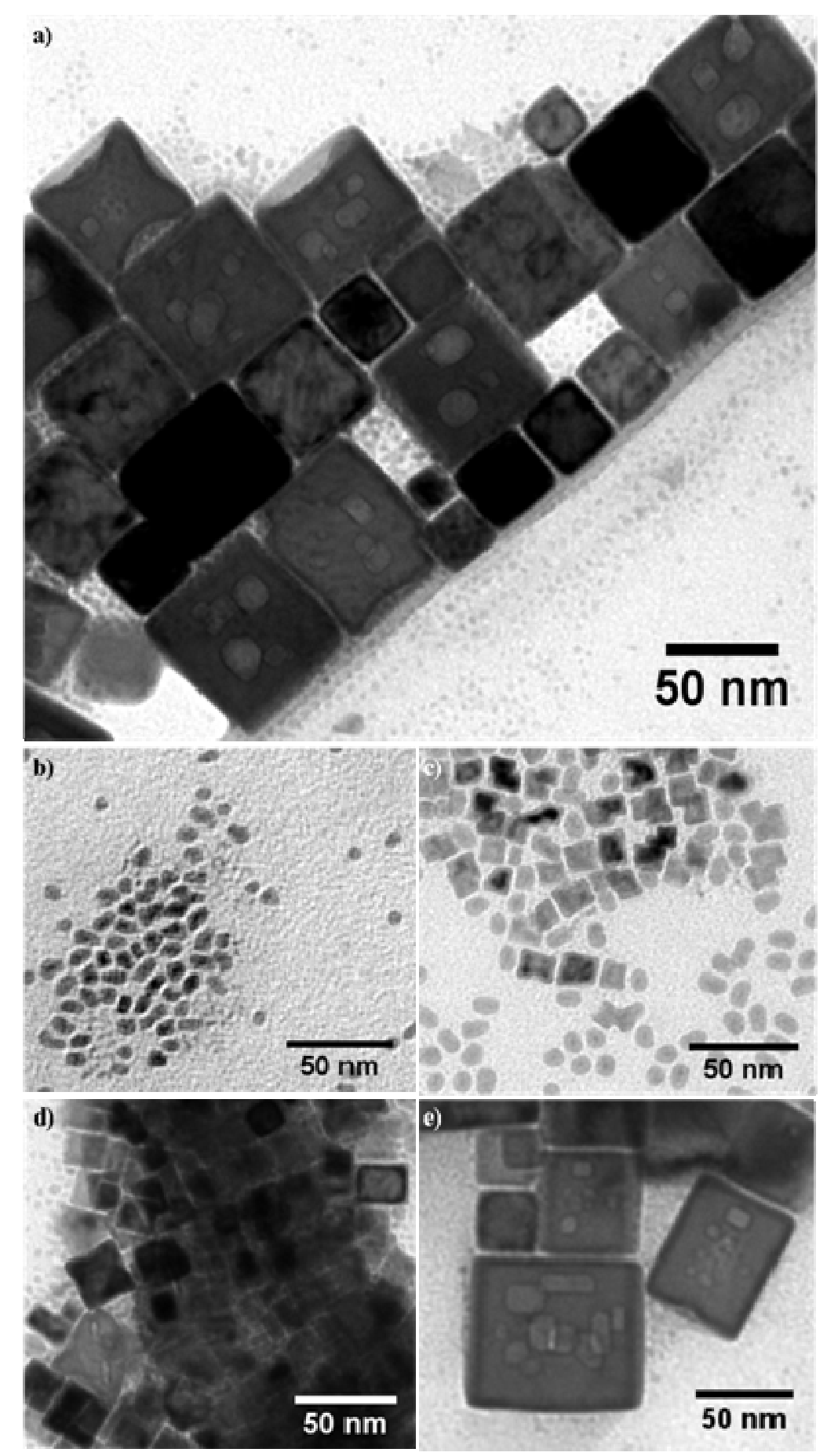

Figure 1 


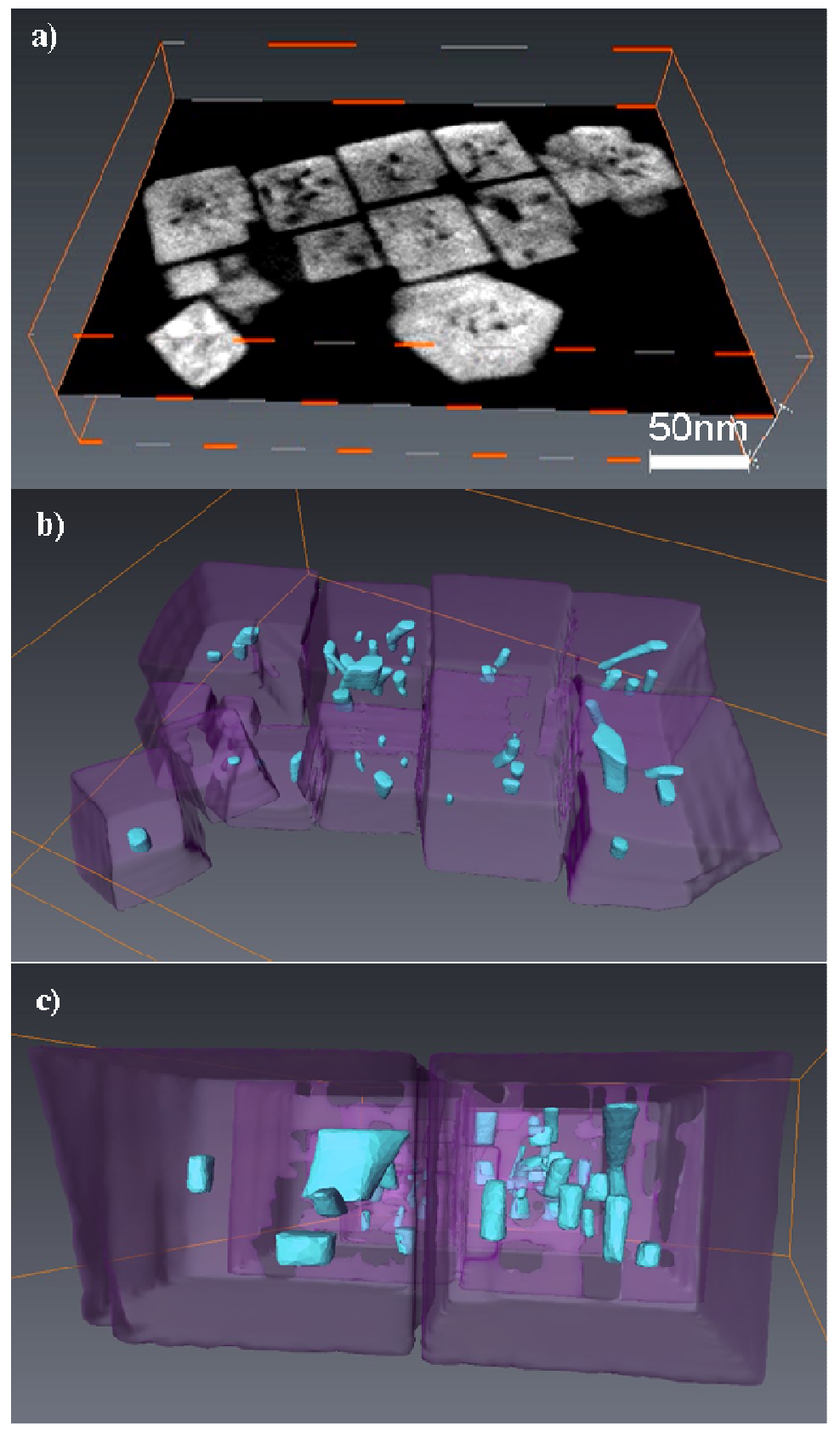

Figure 2 

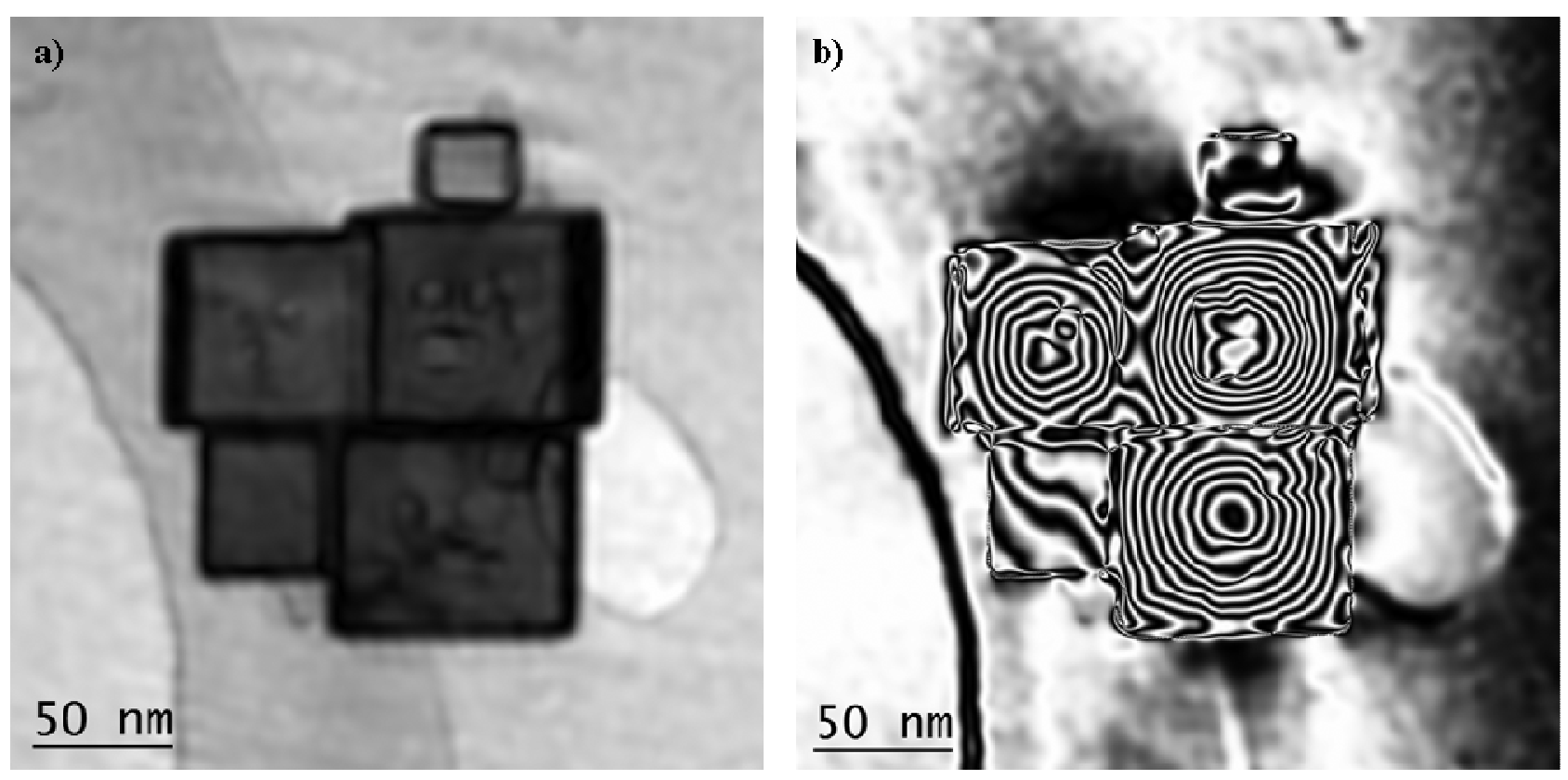

c)

d)
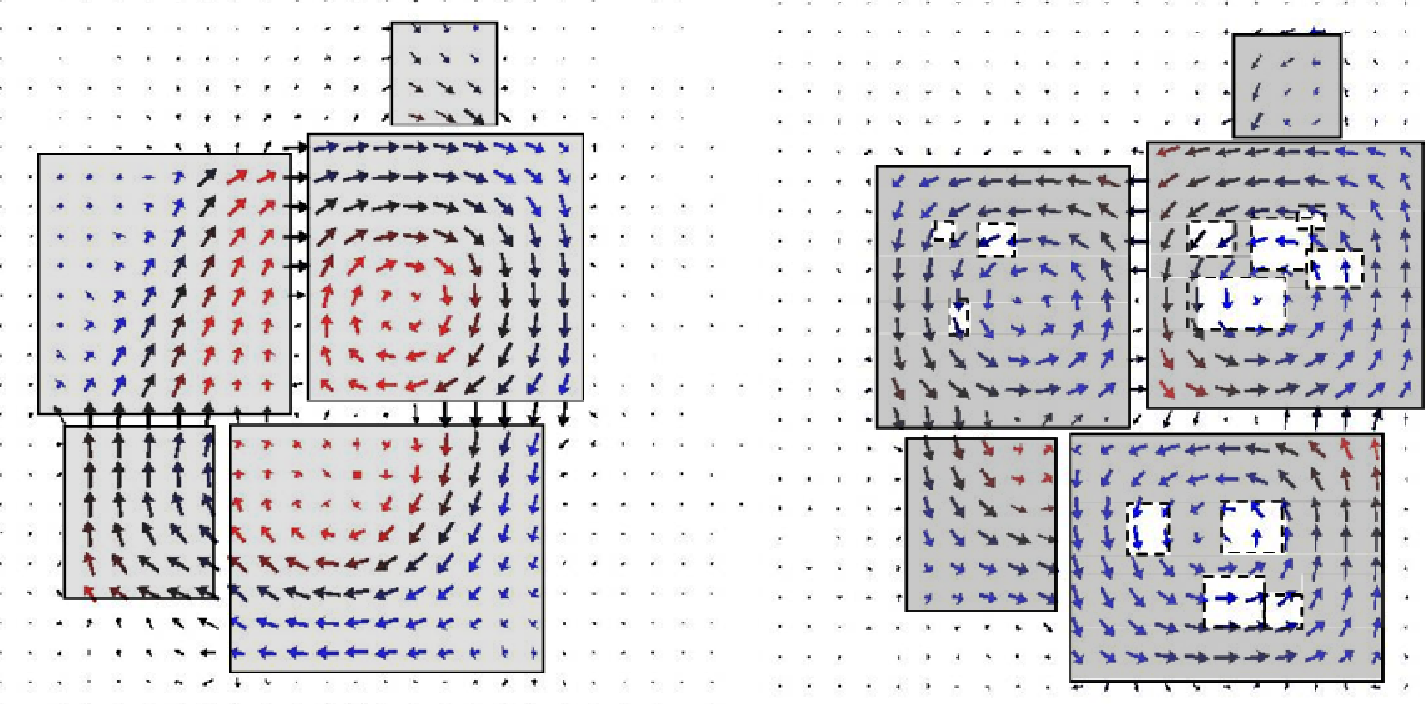

Figure 3 


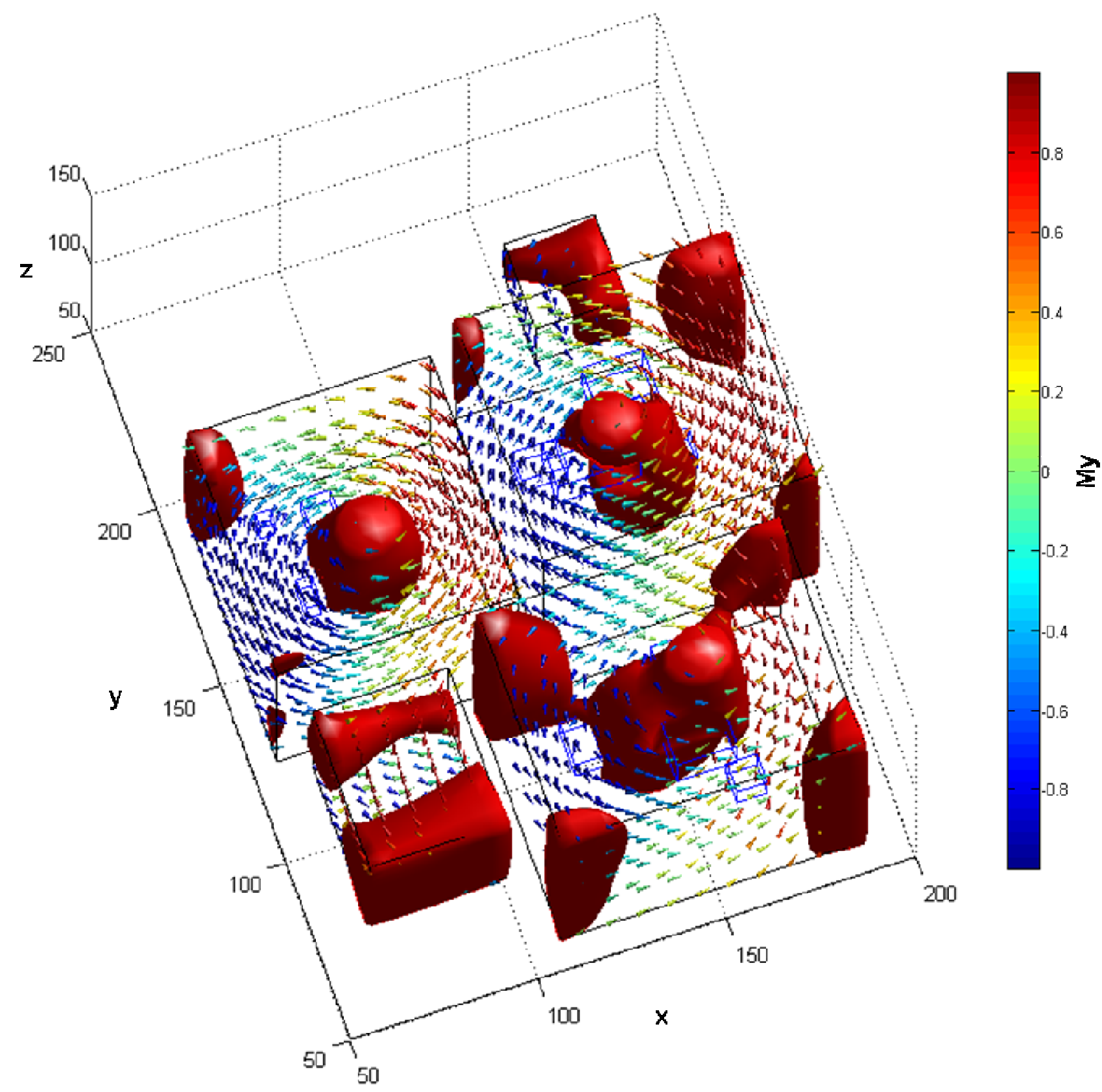

Figure 4 


\section{FIGURE LEGENDS}

Figure 1 : TEM images of a) porous nanocubes obtained at $150^{\circ} \mathrm{C}$ under 3 bars of $\mathrm{H}_{2}$ with an acid/amine ratio of 1.5:2 with alkyl chain $\mathrm{C} 12$, and the intermediate nanoparticles observed after b) $2 \mathrm{~h}$, c) $6 \mathrm{~h}, \mathrm{~d}) 24 \mathrm{~h}$ and e) $48 \mathrm{~h}$ of reaction.

Figure $2: 3 \mathrm{D}$ reconstruction of porous nanocubes obtained by electron tomography a) xy-orthoslice through the 3D reconstruction; b) top view and c) lateral view of the 3D reconstruction, pores are represented in blue while external envelope of the cubes are in transparent violet.

Figure 3 : Electron holography and micromagnetic simulations for a compact assembly of $5 \mathrm{Fe}$ nanocubes. a) TEM image reveals the cubic structure and the internal defects within larger cubes. b) Cosinus of the magnetic phase image corresponding to the magnetic contribution to the phase shift with $\pi / 4 \mathrm{rad}$ isophase contours. Note the cylindrical symmetry of the magnetic induction flux line in the three larger cubes. Micromagnetic simulation (with bulk Ms) of the in-plane induction (the location and the number of arrows are not related with the discretization points) for c) defect-free objects (visualized in gray) and d) porous cubes. Dashed white boxes are a guide to the eye to indicate the lateral extent and the in-plane position of the defects.

Figure 4: 3D Visualisation of the magnetic moment within an assembly of $5 \mathrm{Fe}$ nanocubes. The presence of defects within the three larger cubes is represented with blue boxes. The red surface displays the surface of spins with $M z=0.6$ (we reduced the number of arrows for a better visualisation). 


\section{REFERENCES}

[1] Sun, S.; Murray, C. B.; Weller, D., Folks, L., Moser, A., Monodisperse FePt nanoparticles and ferromagnetic FePt nanocrystal superlattices, Science 2000, 287, 1989-1992

[2] Wetz, F.; Soulantica, K.; Respaud, M.; Falqui, A.; Chaudret, B. Synthesis and magnetic properties of Co nanorod superlattices. Mater. Sci. Eng. C. 2007, 27, 1162-1166

[3] Lau, J. W.; Shaw, J. M. Magnetic nanostructures for advanced technologies: fabrication, metrology and challenges. J. Phys. D: Appl. Phys. 2011, 44, 303001 (2011)

[4] Lim, F.; Wilson, B.; Wood R. Analysis of shringle-write readback using magnetic-force microscopy, IEEE Trans. Magn. 2010, 46, 1548-1551

[5] Gao, J. ; Gu, H.; Xu, B. Multifunctional magnetic nanoparticles : design, synthesis and biomedical applications, Acc. Chem. Res. 2009, 42, 1097-1107

[6] Bouchard, L.-S. et al. Picomolar sensitivity MRI and photoacoustic imaging of cobalt nanoparticles, Proc. Natl. Acad. Sci. 2009, 106, 4085-4089

[7] Lacroix, L.-M.; Ho, D.; Sun, S. Magnetic Nanoparticles as Both Imaging Probes and Therapeutic Agents, Curr. Top. Med.Chem. 2010, 10, 1184-1197

[8] B. Mehdaoui et al. Optimal size of nanoparticles for magnetic hyperthermia: a combined theoretical and experimental study, Adv. Funct. Mater. 2011, 21, 4573-4582

[9] Lu, A.-H.; Salabas, E. L.; Schüth, F. Magnetic nanoparticles: synthesis, protection, functionalization, and application. Angew. Chem. Int. Ed. 2007, 46, 1222-1244

[10] Jeong, U.; Teng, X.; Wang, Y.; Yang, H.; Xia, Y. Superparamagnetic colloids: controlled synthesis and niche applications, Adv. Mater. 2007, 19, 33-60 
[11] Rave, W.; Fabian, K.; Hubert, A. Magnetic states of small cubic particles with uniaxial anisotropy. J. Magn. Magn. Mater. 1998, 190, 332-348

[12] Miltat, J.; Thiaville, A. vortex cores—smaller than small. Science 2002, 298, 555

[13] Van Waeyenberge, B. et al. Magnetic vortex core reversal by excitation with short bursts of an alternating field. Nature 2006, 444, 461-464

[14] Keavney, D. J.; Cheng, X. M.; Buchanan, K. S. Polarity reversal of a magnetic vortex core by a unipolar, nonresonant in-plane pulsed magnetic field. Appl. Phys. Lett. 2009, 94, 172506

[15]Choi Y.-S. et al. Out-of-plane current controlled switching of the fourfold degenerate state of a magnetic vortex in soft magnetic nanodots. Appl. Phys. Lett. 96, 072507 (2010)

[16] Yu, X. W. et al. Images of spin-torque-driven magnetic nano-oscillator, Phys. Rev. Lett. 2011, 106,167202

[17] Dussaux, A. et al. Large microwave generation from D.C. driven magnetic vortex oscillators in magnetic tunnel junctions, Nat. Commun. 2010, 1, 8

[18] Vogel, A. et al. Coupled vortex oscillations in spatially separated permalloy squares, Phys. Rev. Lett. 2011, 106, 137201

[19] Yang, Y.; Liu, X.-L.; Yi, J.-B.; Yang, Y.; Fan, H.-M.; Ding, J. Stable vortex magnetite nanorings colloid: micromagnetic simulation and experimental demonstration, J. Appl. Phys. 2012, 111, 044303

[20] Snoeck, E. et al. Magnetic configurations of $30 \mathrm{~nm}$ iron nanocubes studied by electron holography. Nano Lett. 2008, 8, 4293-4298

[21] Rahm, M.; Biberger, J.; Umansky, V.; Weiss, D. Vortex pinning at individual defects in magnetic nanodisks. J. Appl. Phys. 2003, 93, 7429-7431 
[22] Goll, D.; Berkowitz, A. E.; Bertram, H. N. Critical sizes for ferromagnetic spherical hollow nanoparticles. Phys. Rev. B 2004, 70, 184432

[23] Uhling et al. Shifting and pinning of a magnetic vortex core in a permalloy dot by a magnetic field. Phys. Rev. Lett. 2005, 95, 237205

[24] Peng, S.; Sun, S. Synthesis and characterization of monodisperse hollow $\mathrm{Fe}_{3} \mathrm{O}_{4}$ nanoparticles. Angew. Chem. Int. Ed. 2007, 46, 4155-4158

[25] Lacroix, L.-M.; Lachaize, S.; Falqui, A.; Respaud, M.; Chaudret, B. Iron nanoparticle growth in organic superstructures. J. Am. Chem. Soc. 2009, 131, 549-557

[26] Huo, Z.; Tsung, C.-K.; Huang, W.; Zhang, X.; Yang, P. Sub-two nanometer single crystal Au nanowires. Nano Lett. 2008, 8, 2041-2044

[27] Lu, X.; Yavuz, M. S.; Tuan, H.-Y.; Korgel, B. A.; Xia, Y. Ultrathin gold nanowires can be obtained by reducing polymeric strands of Oleylamine-AuCl complexes formed via aurophilic interaction. J. Am. Chem. Soc. 2008, 130, 8900-8901

[28] Rasband, W.S., ImageJ, U. S. National Institutes of Health, Bethesda, Maryland, USA, http://rsb.info.nih.gov/ij/, 1997-2007.

[29] Kawase, N., Kato, M., Nishioka, H., Jinnai, H. Transmission electron microtomography without the "missing wedge" for quantitative structural analysis. Ultramicroscopy 107, 8-15 (2007)

[30] Donahue M. J. and Porter D. G. OOMMF User's Guide, Version 1.0, Interagency Report 1999, NISTIR 6376, National Institute of Standards and Technology, Gaithersburg, MD. We used version 1.2a3 of this public code that can be found at the URL http://math.nist.gov/oommf/. 
[31] Lacroix L.-M. et al. Ultrasmall iron nanoparticles: Effect of size reduction on anisotropy and magnetization. J. Appl. Phys. 2008, 103, 07D521

[32] Midgley, P. A.; Dunin-Borkowski, R. E. Electron tomography and holography in materials science. Nature Mater. 2009, 8, 271-280

[33] Biermans, E.; Molina, L.; Batenburg, K. J.; Bals, S.; Van Tendeloo, G. Measuring porosity at the nanoscale by quantitative electron tomography. Nano Lett. 2010, 10, 5014-5019

[34] Thomas, J. M.; Simpson, E. T.; Kasama, T.; Dunin-Borkowski, R. E. Electron holography for the study of magnetic nanomaterials. Acc. Chem. Res. 2008, 41, 665-674

[35] Donahue M. J. and Porter D. G. OOMMF User's Guide, Version 1.0, Interagency Report 1999, NISTIR 6376, National Institute of Standards and Technology, Gaithersburg, MD. We used version $1.2 \mathrm{a} 3$ of this public code that can be found at the URL http://math.nist.gov/oommf/.

[36] Liu, Y.; Du, A. Arrangement effects of triangular defects on magnetization reversal process in a permalloy dot. J. Magn. Magn. Mater. 2011, 323, 461-464

[37] Vaz, C. A. F.; Athanasiou, C.; Bland, J. A. C.; Rowlands, G. Energetics of magnetic ring and disk elements: uniform versus vortex state, Phys. Rev. B 2006, 73, 054411 